\title{
Patterns of Local-regional Failures after Adjuvant Chemotherapy in Resected Pancreatic Cancer without adjuvant radiation therapy: Implication for Irradiation Volumes Design
}

Shengguang Zhao

Shanghai Jiao Tong University

Youlia Kirova

Institut Curie

Wei-Xiang Qi ( $\square$ qiweixiang1113@163.com )

ruijin hospital, shanghai jiaotong university

Jiayi Chen

Shanghai Jiao Tong University Medical School Affiliated Ruijin Hospital

Research

Keywords: pancreatic adenocarcinoma, local-regional recurrence pattern, adjuvant chemotherapy, adjuvant radiotherapy, the target volume, competing risk analysis

Posted Date: February 17th, 2020

DOI: https://doi.org/10.21203/rs.2.23761/v1

License: (c) (i) This work is licensed under a Creative Commons Attribution 4.0 International License.

Read Full License 


\section{Abstract}

In the present study, we aimed to assess the patterns of recurrence in pancreatic cancer patients, following surgical resection and adjuvant chemotherapy in the absence of adjuvant radiation , for optimal irradiation volumes design encompassing the majority of local recurrences. Isolated local regional recurrence (LRR) was defined as LR (local recurrence) and/or regional recurrence (RR) without distant metastasis (DM). The Fine and Gray competing risks regression analysis was performed to identified prognostic factors associated with isolated LRR. A total of 85 male and 29 female patients were included, 95 (83.3\%) of the 114 patients had recurred. Most patients recurred at LRR $(n=36,37.9 \%)$, and isolated LR was seen in 23 patients(24.2\%). The median time between the end of the treatment and initial recurrence was 9.8 months (2.2-71). Competing risks analysis demonstrated that surgery combined with vascular resection (HR 2.44, 95\% Cl: $1.40-4.27, \mathrm{p}=0.002$ ) and $\mathrm{R} 1$ resection status (HR 3.96, 95\% Cl: 2.23-7.02, $p<0.0001$ ) significantly increased the risk of developing isolated LRR. The most common site of specific LRR were observed in the lymph node regions (LN) No. $14 p(30.5 \%)$, followed by pancreas or tumor bed (23.7\%), LN No.16b1 (23.7\%), celiac artery (22\%), and superior mesenteric artery (22\%). In conclusion, the risk of developing isolated LRR in patients with PDAC who underwent curative resection and adjuvant treatment remains high, especially for patients received surgery combined with vascular resection or R1 resection. Adjuvant radiotherapy for tumor bed/vessels and involved regional nodes including partially the region No.16 is recommended for this patient population.

\section{Introduction}

Although pancreatic ductal adenocarcinoma (PDAC) only accounts for $2.5 \%$ (458,918 newly diagnosed PDAC) of all new cancer cases worldwide in 2018, it is one of the most fatal malignancies, with a total of 432,242 patients died annually[1]. Surgical resection with negative margins (R0) is the only potentially curative treatment for PDAC, but only a minority of these patients (15-20\%) are eligible for resection at the initial diagnosis. For patients after microscopically pathologically complete removal of tumor (R0), the vast majority of these patients would relapse within 2 years. CONKO-001 trials, the first phase III randomized trials investigating the effects of adjuvant gemcitabine chemotherapy in resected PDAC, demonstrated that the use of adjuvant gemcitabine for 6 months compared with observation alone resulted in increased overall survival as well as disease-free survival, which supporting the use of adjuvant chemotherapy in this settings [2,3]. However, the CONKO-005 trial investigating the combination of chemotherapy and a targeted therapy in the adjuvant treatment of PDAC found that gemcitabine combined with erlotinib falied to improve disease-free survival (11.4 vs. 11.4 months) or overall survival (24.5 vs. 26.5 months) over gemcitabine alone, although there was a trend toward long-term survival in favor of combination therapy (5-year OS: $25 \%$ vs. $20 \%$ )[4]. While another ESPAC-4 trial found that the adjuvant combination of gemcitabine and capecitabine was superior to gemcitabine alone group (median OS, 28 versus 25.5, hazard ratio 0.82 [95\% $\mathrm{Cl} 0.68-0.98], \mathrm{p}=0.032$ )[5]. Based on these highquality clinical evidence, curative surgery resection in combination with adjuvant chemotherapy is regared as the standard treatment regimen for resectable PDAC [6, 7]. However, the long-term survival of 
this patient population remains poor, with 5-year overall survival of $25 \%$. Clearly, more efficient treatments are still needed to optimizing adjuvant strategies in this settings.

In the ESPAC-4 trial[5], approximately 50\% resected PDAC patients received adjuvant chemotherapy would develop local recurrence, which is the most common site of relapse. In addition, nearly $25 \%$ patients in CONKO-005 trial would develop isolated local recurrence. As a result, the addition of radiotherapy to adjuvant therapy in PDAC might reduce local recurrence and improve survial. Indeeds, several randomized clinical trials have been conducted to investigate the role of adjuvant chemoradiotherapy in PDAC patients, but the results is controversial [8-12]. One possible explanation for this finding is that there is no agreement on the definition of irradiation volumes and the irradiation strategies vary among these trials. In addition, there is no published studies to optimizing the irradiation volumes based on the recurrence patterns of PDAC patients. Therefore, we use the Fine and Gray competing risks regression analysis to clearly define the risk factors for LRR and optimize the irradiation volumes in resected PDAC after adjuvant chemotherapy.

\section{Patients And Methods}

We used an institutional pancreatic tumor registry to identify all patients who received adjuvant chemotherapy between January 2008 and December 2013. Pancreatoduodenectomy or distal pancreatectomy was performed according to the tumor location. The staging was established according to AJCC TNM classification, $7^{\text {th }}$ edition. Adjuvant chemotherapy regimens were mainly based on gemcitabine (G), as following: ( $1 \mathrm{~g} / \mathrm{m} 2 \mathrm{~d} \mathrm{1,} 8$ and 15 every 4 weeks) for 6 months. We excluded the patients who received less than 2 courses of adjuvant chemotherapy or those who received radiotherapy before. Clinical follow-up and enhanced computed tomography (CT) scan were performed every 2-3 months during adjuvant chemotherapy and after completion of therapy. Patients without evidence of recurrent disease in 2 years after surgical resection were evaluated every 6 months. The development of a new low-density mass was considered as tumor recurrence by scheduled CT scan. Recurrent lymph nodes were defined as short axis $>1 \mathrm{~cm}$, abnormal round morphology, heterogeneity, or central necrosis[13]. The recurrence were confirmed by PET-CT or multidisciplinary review if necessary. Tissue biopsy confirmation was rarely obtained. The present study procedures were approved by the Ethical Committee of Rui Jin Hospital affiliated medicine school of Shanghai Jiao Tong University, all subjects provided written informed consent.

Recurrence patterns were categorized as local, regional recurrence or distant metastasis(LR,RR,DM)[14]. LR was defined as recurrence in the region of tumor bed (including the remnant pancreatic) and involved local vessel (the superior mesenteric artery (SMA), celiac trunk (CT), portal vein (PV), inferior vena cava (IVC), etc. RR was defined as recurrence in lymph nodes within regional pancreatic lymph node drainage region and the retroperitoneal region. Lymph node station numbers was adopted by the Japan Pancreas Society (JPS) staging system to more accurately describe the location of lymph nodes[15, 16]. DM was defined as recurrence in the liver, lungs, omentum, or other distant organs (include ascites). The isolated LRR includes LR, RR, or simultaneous LR and RR without DM. Any DM included DM only. Whether or not 
there is LR or RR. Only first sites and time of initial recurrence on imaging were documented.After the diagnosis of recurrence, the treatment was determined by the multidisciplinary team according to patient's performance status and disease progression level. Second-line systemic chemotherapy or chemo radiotherapy regimens were typically employed.

\section{Statistical analysis}

Overall survival (OS) was defined as the time from the date of surgery to death, censoring patients who were alive on the date of last follow-up. Progression-free survival (PFS) was measured from operation date to whichever the date of the first recurrence or of the last follow-up. Descriptive statistics were used to summarize the study data. Continuous variables were expressed as the median and range, and categorical variables as a number and percentage. We used the Kaplan-Meier method to analyze survival time and the Log-rank test to compare the isolated LRR and any DM group. Cox proportional hazard model is used to identify significant prognostic factors of PFS and isolated LRR. For any DM is a competing risk event which prevent isolated LRR from occurring and the Fine-Gray's model were used to further assess the effect of patient demographic, clinical, pathologic factors on isolated LRR. Statistical analyses were performed using IBM SPSS Statistics for Mac, version 23.0. (SPSS, Chicago, IL). FineGray's model analysis is performed in R 3.4.3 using the crr package. Statistical significance established at $\mathrm{P}<0.05$, all statistical tests were 2-sided.

\section{Results}

\section{Patients' outcome}

Between January 2008 and February 2013, a total of 310 patients with pancreatic cancer received chemotherapy. After excluding patients without surgery $(n=119)$ or without radical resection $(n=16)$, other histology than adenocarcinoma $(n=18)$, as well as those with intraoperative radiotherapy $(n=4)$, and those receiving less than 2 courses of chemotherapy $(n=16)$ or developing recurrence before adjuvant chemotherapy $(n=23)$, we studied 114 patients with PDAC treated with curative surgery plus adjuvant chemotherapy considered eligible for investigating the patterns of the failure.

\section{Recurrence Incidence}

After a median follow-up of 42 months (range, 3-92.3), 95 of 114 patients (83.3\%) developed recurrence. LR occurred in 23 patients (24.2\%), RR in 20 (21.1\%), DM in 16 (16.8\%), LR with RR in 16 cases (16.8\%), RR with DM in 12 patients (12.6\%), LR with DM in 1 case (1.1\%), LR and RR with DM in 7 patients (7.4\%). Figure 1 showed the rate of isolated LRR and any DM per 6-month follow-up period after surgery . The incidence of isolated LRR and any DM was $31.6 \%$, and $23.7 \%$ in the first year, $14 \%$ and $7 \%$ in the second year, $6.1 \%$ and $0.9 \%$ in the third year respectively.

\section{Survival Analysis}


The median time between the end of the treatment and initial recurrence was 9.8 months (range, 2.2-71). Of all 114 patients, median OS time was 30.8 months (95\% confidence interval [Cl] 26.8-34.9) and the median PFS was 11 months (95\% Cl 9-13)(figure 2a and 2b). In the LRR group, the OS was significantly longer (median OS, 31.5 months; $95 \% \mathrm{Cl}, 28.2-34.8$ ) than in patients presented with DM (median OS, 14.6 months $)(p<0.0001)$. The 2-year and 5-year survival rate in isolated LRR group was $75.9 \%$ and $11.1 \%$ in comparison with DM group: $30.5 \%$ and $0 \%$.

The median PFS in isolated LRR group was 10.2 months (95\% $\mathrm{Cl}, 8.8-11.7)$ significantly longer than in any DM group 7.1months $(95 \% \mathrm{Cl}, 4.6-9.6)(p=0.019)$. Actuarial 1-year and 2-year rates of PFS of patients with isolated LRR was $42.3 \%$ and $14.7 \%$, as compared with $25.0 \%$ and $2.8 \%$ in patients with any DM. (Figure 2c and 2d)

\section{Prognostic Factors for Isolated LRR and PFS}

The univariable and multivariable analyses for risk factors for PFS and isolated LRR are shown in Table 1 and Table 2. Positive resection margins (HR 1.76, 95\% Cl: $1.26-3.63, p=0.005)$ and poor differentiation (HR 2.07, 95\% Cl: $1.31-3.28, p=0.002$ ) were correlated with worse PFS. Adjuvant treatment courses more than 6 cycles was associated with improved PFS (HR 0.51, 95\% Cl: $0.33-0.80, p=0.003$ ). The predictive factors of isolated LRR, obtained by Cox proportional hazard model univariate and multivariate analysis, was only R1 resection status (HR 3.86, 95\% Cl: 2.09-7.16, $p<0.0001$ ). Considering the competitive risk event, we adopted competing risk analysis to adjust the risk factor assessment. The analysis demonstrated that surgery combined with vascular resection (HR 2.44, 95\% $\mathrm{Cl}: 1.40-4.27, p=0.002$ ) and $\mathrm{R} 1$ resection status (HR 3.96, 95\% Cl: 2.23-7.02, $p<0.0001$ ) increased risk of isolated LRR. Cumulative incidence curves for patients presented with or without vascular resection are statistically different for isolated LRR $(p=0.025)$ and any DM $(p=0.021)$, the cumulative incidence curves for resection status were not significantly different for any DM $(p=0.1)$ but are highly significant for $\operatorname{LRR}(p<0.0001$, figure 3$)$.

\section{Recurrence sites}

In the 59 patients with only LRR, the major recurrent sites were No.14p lymph nodes(30.5\%), remnant pancreas or tumor bed(23.7\%), No.16b1 lymph nodes (23.7\%),CA(22.0\%), SMA(22.0\%), and No. $9(20.3 \%), 12 p(15.3 \%), 14 d(13.6 \%)$ lymph nodes. In the any DM of patients also had some local- regional recurrence, mainly of No.16b1(33.3\%), 12p(19.4\%), 16a2(19.4\%), 14p(16.7\%),14d(16.7\%) and No.

$9(16.7 \%)$ lymph nodes, SMA(16.7\%), CA(11.1\%).For all patients with relapse, the susceptible involved sites are No.16b1(27.4\%), 14p(25.3\%) lymph nodes, SMA(20.0\%), No. 9(18.9\%), CA(17.9\%), $12 \mathrm{p}(16.8 \%)$,remnant pancreas or tumor bed(15.8\%), No.14d(14.7\%), 16a2(10.5\%) lymph nodes (Table 3.)

\section{Discussion}

Although adjuvant chemotherapy in resected PDAC patients can significantly improve overall survival in comparison with observation, the majority of these patients would eventally develop local recurrence with or without distant metastasis. Since not all PDAC patients recur in the same manner, understanding the 
pattern, timing, and implications of recurrence is critical to the planning of adjuvant strategies. In addition, clinicopathologic risk factors for predicting failure patterns could potentially allow individualized and more effective adjuvant therapy strategies $[17,18]$. However, to our best knowledge, there is no published studies to specially investigate the risk predictor factors for isolated LRR in PDCA patients by using competing risk regression model, and the patterns of local regional recurrence of lymph node are remains undetermined. As a result, we conduct the present study to clearly define the patterns of recurrence and identify the risk factors for isolated LRR, in order to optimize the irradiation volume and perform individualized treatments.

A total of 114 patients are included for analysis. In consistent with previously published data, 95 of 114 patients (83.3\%) develop recurrence after a median follow-up of 42 months, and the isolated local recurrence is observed in 23 patients(24.2\%). Previously published data also demonstrated that the prognosis of patients with local recurrence is better than that of distant metastasis [19-21]. In the present study, we also found that the interval of patients developing isolated LRR is significantly longer than those recurred with any DM after the adjuvant chemotherapy. This may be explained for this finding is that micro-metastasis might exist before the surgical resection of primary tumor [22-24]. As a result, local treatment options such as re-resection and radiotherapy could be considered to for those isolated LRR patients to improve the survival.

Adjuvant chemotherapy can also improve the local control rate[25, 26]. However, with the completion of the chemotherapy, it is hypothesized that the local-regional problem will emerge as the main challenge. In consistent with this hypothesis, our study demonstrates that the incidence of isolated LRR is significantly higher than that of DM after completion of adjuvant chemotherapy. Although most patients with PDAC would die from distant metastases, the mortality after local recurrence is still $20 \%$. And the natural evolution is the development of hepatic metastases after the local recurrence[27]. Therefore, the avoiding of the LRR becoming a secondary source of DM, it may be an important reason for application radiochemotherapy following adjuvant chemotherapy after curative surgery.

In order to reduce post-operative recurrence, increasingly radical surgery with extended lymph node dissection and combined with vascular resection has been explored but the efficacy is debated [28-31]. The combination with vascular resection improved the prognosis of patients in comparison with not resected tumors [32,33]. This surgery is associated with higher probability of positive surgical margins [34-36]. Furthermore, tumor involving arterial structures (especially T4 tumor) recur rapidly, even after an apparent R0 resection[36]. In our series, the vascular resection is associated with a higher risk of isolated LRR as previously reported in the literature [37, 38]. However, this phenomenon is masked by the decline of distant metastasis rate after vascular resection when analyzed by the cox regression model. There are two types of vascular involvement after vascular resection: neoplastic vessel invasion on histopathology and inflammatory adhesion[35]. Mierke et. al. reported that patients who had inflammatory adhesion has a lower distant metastasis rate than those who had tumor infiltration and those without vascular resection[39]. Thus, the involvement of peripancreatic vessels seems to be an indicator of unfavorable 
tumor topography [40]. One of the limitations of our retrospective study is that it can not provide the proportion of inflammatory adhesion in those patients with vascular resection.

Positive surgical margin (R1 resection) is a major adverse prognostic factor of disease-free survival(DFS) for resectable PDAC[27, 41, 42] though lack of consensus on definition of margin status[43]. The definition of positive margin in our institution is the directed involvement of tumor at any of the edges of the resection specimen. In our study, R1 resection rate was about $20 \%$ and R1 status was a significant predictor of isolated LRR but not significantly increase rates of any DM in PDAC patients after received adjuvant chemotherapy. Systemic chemotherapy was still effective for decreasing recurrence in R1 patients, with small benefit as previously reported [44]. Our study shows that after chemotherapy, localregional problems become the focus of attention in patients with R1 resection. Therefore the idea of this work is that the loco regional radiotherapy with careful volume definition and delineation after attentive analysis of the patterns could be alternative for this population of patients. We have shown that the remnant pancreas or tumor bed was the common site of recurrence $(23.7 \%)$ and it must be included in the target volume of irradiation. Miyazaki et al. reported 13/67(19\%) patients with isolated local recurrence in the remnant pancreas[45]. Of the many major vessels near the pancreas, the most likely to relapse is the SMA and the CA. This occurs not only due to their features easy to directly involved from the primary tumor but also the practical difficulties for surgical clearance, and radiation oncologists have attached vital importance to them from the era of two dimensional radiotherapy treatment to modern radiotherapy[46-48].

So far, several studies optimizing the adjuvant radiotherapy field for pancreatic cancer have been published [47-49]. However, these studies generally use the Cox regression model to identify the risk factors for LRR. In the present study, we use the competing risks regression model to define the risk factors and comprehensively investigate the recurrence patterns of regional lymph nodes. We believe that the understanding based on vascular anatomy may make easier to delineate, however, for the analysis of lymph node metastasis, there is a lack of uniformity. Thus, we used JPS stage system to study the recurrence of the peritoneal lymph nodes, and we find that nodal metastasis is commonly involved in No.14p, No.16b1, No. 9 and No. 12p. The outcome is consistent with the pathological results after surgery[50], besides the lymph nodes around the pancreas, the regional lymph nodes are mainly involved in group No.9, No.12 group, No.14 group. Furthermore, para-aortic lymph node (No.16) metastasis, regarded as distant metastasis rather than regional lymph node and classified as pM1 stage, is found in 37 of 114 patients $(32.4 \%)$ after adjuvant chemotherapy, which approximately involved $14-18 \%$ at the time of resection[51-54]. No.16 is classified into four groups according to JPS system $(16 \mathrm{a} 1,16 \mathrm{a} 2,16 \mathrm{~b} 1$, $16 \mathrm{~b} 2)$. In the present study, No.16b1 is frequently involved (27.4\% of all recurrences). Especially, in any DM group, No.16b1 is involved more frequently with the recurrence of tumor bed apparently decreased (2.8\%) compared to the isolated group. A recent meta-analysis also confirmed that the status of lymph node para-aortic (No.16) metastases had a significant prognostic value[53]. Our study suggests that the optimal radiotherapy volume may include not only the tumor bed and vessels but also involved regional nodes including partially the region No.16 (Figure. 4). 
The findings of present study should be interpreted with some caution due to the following limitations: (1) first of all, our study is a single institute retrospective studies, and selection bias is unavoidable, the true impact of adjuvant chemotherapy on recurrence patterns of pancreatic cancer could be underestimated in this study; (2) secondly, the sample size for our study might be relatively small to evaluate all patterns of recurrences, although the median follow-up time is longer than three years. The stength of this study is that we only include resectable pancreatic cancer received with adjuvant chemotherapy, and patients, who initially diagnosed with " borderline resectable" pancreatic cancer and received neoadjuvant therapy, are excluded for analysis, while this patient cohort is generally included for analysis in several published studies [11,33-36], as a result, the clinical heterogeneities of the present is relative small than previous publication.

\section{Conclusion}

In conclusion, the risk of developing isolated LRR in patients with PDAC who underwent curative resection and adjuvant treatment remains high, especially for patients received surgery combined with vascular resection or R1 resection. Adjuvant radiotherapy for tumor bed/vessels and involved regional nodes including partially the region No.16 is recommended for this patient population.

\section{Abbreviations}

$L R R$, local regional recurrence; $R R$, regional recurrence; $D M$, distant metastasis; $L R$, local recurrence; $L N$, lymph node; PDAC, pancreatic ductal adenocarcinoma; G, gemcitabine; CT, computed tomography; SMA, superior mesenteric artery; CT, celiac trunk; PV, portal vein, IVC, inferior vena cava; OS, Overall survival; PFS, Progression-free survival;

\section{Declarations}

\section{Ethics approval and consent to participate}

The present study procedures were approved by the Ethical Committee of Rui Jin Hospital affiliated medicine school of Shanghai Jiao Tong University. All procedures performed were in accordance with the ethical standards of the institutional and/or national research committee and with the 1964 Helsinki Declaration and its later amendments or comparable ethical standards. Informed consent was obtained from all participants included in the study.

\section{Consent for publication}

Not applicable.

\section{Availability of supporting data}

All data included in this study are available upon request by contact with the corresponding author. 
Competing interests

The authors declare that they have no competing interests.

Acknowledgement: none

Funding: none

Authors' information

Conceived and designed the experiments: Jiayi Chen. Performed the experiments: Shengguang Zhao and Wei-xiang Qi. Analyzed the data: Shengguang Zhao. Contributed reagents/materials/analysis tools: WeiXiang Qi. Wrote the paper: Jiayi Chen and Youlia Kirova.

\section{References}

1. Bray F, Ferlay J, Soerjomataram I, Siegel RL, Torre LA, Jemal A: Global cancer statistics 2018: GLOBOCAN estimates of incidence and mortality worldwide for 36 cancers in 185 countries. $C A$ Cancer J Clin 2018, 68(6):394-424.

2. Neoptolemos JP, Dunn JA, Stocken DD, Almond J, Link K, Beger H, Bassi C, Falconi M, Pederzoli P, Dervenis $\mathrm{C}$ et al: Adjuvant chemoradiotherapy and chemotherapy in resectable pancreatic cancer: a randomised controlled trial. Lancet 2001, 358(9293):1576-1585.

3. Oettle H, Neuhaus P, Hochhaus A, Hartmann JT, Gellert K, Ridwelski K, Niedergethmann M, Zulke C, Fahlke J, Arning MB et al: Adjuvant chemotherapy with gemcitabine and long-term outcomes among patients with resected pancreatic cancer: the CONKO-001 randomized trial. JAMA 2013, 310(14):1473-1481.

4. Sinn M, Bahra M, Liersch T, Gellert K, Messmann H, Bechstein W, Waldschmidt D, Jacobasch L, Wilhelm M, Rau BM et al: CONKO-005: Adjuvant Chemotherapy With Gemcitabine Plus Erlotinib Versus Gemcitabine Alone in Patients After RO Resection of Pancreatic Cancer: A Multicenter Randomized Phase III Trial. J Clin Oncol 2017, 35(29):3330-3337.

5. Neoptolemos JP, Palmer DH, Ghaneh P, Psarelli EE, Valle JW, Halloran CM, Faluyi O, O'Reilly DA, Cunningham $\mathrm{D}$, Wadsley $\mathrm{J}$ et al: Comparison of adjuvant gemcitabine and capecitabine with gemcitabine monotherapy in patients with resected pancreatic cancer (ESPAC-4): a multicentre, open-label, randomised, phase 3 trial. Lancet 2017, 389(10073):1011-1024.

6. Pisters PW, Wolff RA, Crane $\mathrm{CH}$, Evans DB: Combined-modality treatment for operable pancreatic adenocarcinoma. Oncology (Williston Park) 2005, 19(3):393-404, 409; discussion 409-310, 412-396.

7. Bilimoria KY, Bentrem DJ, Ko CY, Tomlinson JS, Stewart AK, Winchester DP, Talamonti MS: Multimodality therapy for pancreatic cancer in the U.S. : utilization, outcomes, and the effect of hospital volume. Cancer 2007, 110(6):1227-1234.

8. Neoptolemos JP, Stocken DD, Friess H, Bassi C, Dunn JA, Hickey H, Beger H, Fernandez-Cruz L, Dervenis $\mathrm{C}$, Lacaine $\mathrm{F}$ et al: A randomized trial of chemoradiotherapy and chemotherapy after 
resection of pancreatic cancer. N Engl J Med 2004, 350(12):1200-1210.

9. Hsu CC, Herman JM, Corsini MM, Winter JM, Callister MD, Haddock MG, Cameron JL, Pawlik TM, Schulick RD, Wolfgang CL et al: Adjuvant chemoradiation for pancreatic adenocarcinoma: the Johns Hopkins Hospital-Mayo Clinic collaborative study. Ann Surg Oncol2010, 17(4):981-990.

10. Van Laethem JL, Hammel P, Mornex F, Azria D, Van Tienhoven G, Vergauwe P, Peeters M, Polus M, Praet $\mathrm{M}$, Mauer $\mathrm{M}$ et al: Adjuvant gemcitabine alone versus gemcitabine-based chemoradiotherapy after curative resection for pancreatic cancer: a randomized EORTC-40013-22012/FFCD9203/GERCOR phase II study. Journal of clinical oncology : official journal of the American Society of Clinical Oncology 2010, 28(29):4450-4456.

11. Colbert LE, Hall WA, Nickleach D, Switchenko J, Kooby DA, Liu Y, Gillespie T, Lipscomb J, Kauh J, Landry JC: Chemoradiation therapy sequencing for resected pancreatic adenocarcinoma in the National Cancer Data Base. Cancer 2014.

12. Rutter CE, Park HS, Corso CD, Lester-Coll NH, Mancini BR, Yeboa DN, Johung KL: Addition of radiotherapy to adjuvant chemotherapy is associated with improved overall survival in resected pancreatic adenocarcinoma: An analysis of the National Cancer Data Base. Cancer 2015, 121(23):4141-4149.

13. Van den Broeck A, Sergeant G, Ectors N, Van Steenbergen W, Aerts R, Topal B: Patterns of recurrence after curative resection of pancreatic ductal adenocarcinoma. Eur J Surg Oncol 2009, 35(6):600-604.

14. Al-Hawary MM, Francis IR, Chari ST, Fishman EK, Hough DM, Lu DS, Macari M, Megibow AJ, Miller $\mathrm{FH}$, Mortele $\mathrm{KJ}$ et al: Pancreatic ductal adenocarcinoma radiology reporting template: consensus statement of the Society of Abdominal Radiology and the American Pancreatic Association. Radiology 2014, 270(1):248-260.

15. Kawarada Y, Isaji S: Stage classifications of pancreatic cancer: comparison of the Japanese and UICC classifications and proposal for a new staging system. Union Internationale Contre le Cancer. Pancreas 1998, 16(3):255-264.

16. Raut CP, Tseng JF, Sun CC, Wang H, Wolff RA, Crane CH, Hwang R, Vauthey JN, Abdalla EK, Lee JE et al: Impact of resection status on pattern of failure and survival after pancreaticoduodenectomy for pancreatic adenocarcinoma. Annals of surgery 2007, 246(1):52-60.

17. Yamamoto $\mathrm{T}$, Uchida $\mathrm{Y}$, Terajima H: Clinical impact of margin status on survival and recurrence pattern after curative-intent surgery for pancreatic cancer. Asian J Surg 2019, 42(1):93-99.

18. Groot VP, Gemenetzis G, Blair AB, Ding D, Javed AA, Burkhart RA, Yu J, Borel Rinkes IH, Molenaar IQ, Cameron JL et al: Implications of the Pattern of Disease Recurrence on Survival Following Pancreatectomy for Pancreatic Ductal Adenocarcinoma. Ann Surg Oncol 2018, 25(8):2475-2483.

19. Westerdahl J, Andren-Sandberg A, Ihse I: Recurrence of exocrine pancreatic cancer-local or hepatic? Hepatogastroenterology 1993, 40(4):384-387.

20. Sperti C, Pasquali C, Piccoli A, Pedrazzoli S: Recurrence after resection for ductal adenocarcinoma of the pancreas. World J Surg 1997, 21(2):195-200. 
21. Suenaga M, Fujii T, Kanda M, Takami H, Okumura N, Inokawa Y, Kobayashi D, Tanaka C, Yamada S, Sugimoto $\mathrm{H}$ et al: Pattern of first recurrent lesions in pancreatic cancer: hepatic relapse is associated with dismal prognosis and portal vein invasion. Hepatogastroenterology 2014, 61(134):1756-1761.

22. Campbell PJ, Yachida S, Mudie LJ, Stephens PJ, Pleasance ED, Stebbings LA, Morsberger LA, Latimer C, McLaren S, Lin ML et al: The patterns and dynamics of genomic instability in metastatic pancreatic cancer. Nature 2010, 467(7319):1109-1113.

23. Yachida S, Jones S, Bozic I, Antal T, Leary R, Fu B, Kamiyama M, Hruban RH, Eshleman JR, Nowak MA et al: Distant metastasis occurs late during the genetic evolution of pancreatic cancer. Nature 2010, 467(7319):1114-1117.

24. Maddipati R, Stanger BZ: Pancreatic Cancer Metastases Harbor Evidence of Polyclonality. Cancer Discov 2015, 5(10):1086-1097.

25. Oettle H, Post S, Neuhaus P, Gellert K, Langrehr J, Ridwelski K, Schramm H, Fahlke J, Zuelke C, Burkart $C$ et al: Adjuvant chemotherapy with gemcitabine vs observation in patients undergoing curative-intent resection of pancreatic cancer: a randomized controlled trial. JAMA : the journal of the American Medical Association 2007, 297(3):267-277.

26. Ueno H, Kosuge T, Matsuyama Y, Yamamoto J, Nakao A, Egawa S, Doi R, Monden M, Hatori T, Tanaka $\mathrm{M}$ et al: A randomised phase III trial comparing gemcitabine with surgery-only in patients with resected pancreatic cancer: Japanese Study Group of Adjuvant Therapy for Pancreatic Cancer. Br J Cancer 2009, 101(6):908-915.

27. Hishinuma S, Ogata Y, Tomikawa M, Ozawa I, Hirabayashi K, Igarashi S: Patterns of recurrence after curative resection of pancreatic cancer, based on autopsy findings. Journal of gastrointestinal surgery : official journal of the Society for Surgery of the Alimentary Tract 2006, 10(4):511-518.

28. Pedrazzoli S, DiCarlo V, Dionigi R, Mosca F, Pederzoli P, Pasquali C, Kloppel G, Dhaene K, Michelassi F: Standard versus extended lymphadenectomy associated with pancreatoduodenectomy in the surgical treatment of adenocarcinoma of the head of the pancreas: a multicenter, prospective, randomized study. Lymphadenectomy Study Group. Ann Surg 1998, 228(4):508-517.

29. Nimura Y, Nagino M, Takao S, Takada T, Miyazaki K, Kawarada Y, Miyagawa S, Yamaguchi A, Ishiyama S, Takeda $Y$ et al: Standard versus extended lymphadenectomy in radical pancreatoduodenectomy for ductal adenocarcinoma of the head of the pancreas: long-term results of a Japanese multicenter randomized controlled trial. J Hepatobiliary Pancreat Sci 2012, 19(3):230241.

30. Jang JY, Kang MJ, Heo JS, Choi SH, Choi DW, Park SJ, Han SS, Yoon DS, Yu HC, Kang KJ et al: A prospective randomized controlled study comparing outcomes of standard resection and extended resection, including dissection of the nerve plexus and various lymph nodes, in patients with pancreatic head cancer. Ann Surg 2014, 259(4):656-664.

31. Orci LA, Meyer J, Combescure C, Buhler L, Berney T, Morel P, Toso C: A meta-analysis of extended versus standard lymphadenectomy in patients undergoing pancreatoduodenectomy for pancreatic adenocarcinoma. HPB (Oxford) 2015, 17(7):565-572. 
32. Mollberg N, Rahbari NN, Koch M, Hartwig W, Hoeger Y, Buchler MW, Weitz J: Arterial resection during pancreatectomy for pancreatic cancer: a systematic review and meta-analysis. Ann Surg 2011, 254(6):882-893.

33. Gurusamy KS, Kumar S, Davidson BR, Fusai G: Resection versus other treatments for locally advanced pancreatic cancer. Cochrane Database Syst Rev 2014(2):CD010244.

34. Yu XZ, Li J, Fu DL, Di Y, Yang F, Hao SJ, Jin C: Benefit from synchronous portal-superior mesenteric vein resection during pancreaticoduodenectomy for cancer: a meta-analysis. Eur J Surg Oncol 2014, 40(4):371-378.

35. Giovinazzo F, Turri G, Katz MH, Heaton N, Ahmed I: Meta-analysis of benefits of portal-superior mesenteric vein resection in pancreatic resection for ductal adenocarcinoma. Br J Surg 2016, 103(3):179-191.

36. Song A, Liu F, Wu L, Si X, Zhou Y: Histopathologic tumor invasion of superior mesenteric vein/ portal vein is a poor prognostic indicator in patients with pancreatic ductal adenocarcinoma: results from a systematic review and meta-analysis. Oncotarget 2017, 8(20):32600-32607.

37. Takahashi S, Kinoshita T, Konishi M, Gotohda N, Kato Y, Kinoshita T, Kobayashi T, Mitsunaga S, Nakachi K, Ikeda M: Borderline resectable pancreatic cancer: rationale for multidisciplinary treatment. J Hepatobiliary Pancreat Sci 2011, 18(4):567-574.

38. Wang J, Estrella JS, Peng L, Rashid A, Varadhachary GR, Wang H, Lee JE, Pisters PW, Vauthey JN, Katz $\mathrm{MH}$ et al: Histologic tumor involvement of superior mesenteric vein/portal vein predicts poor prognosis in patients with stage II pancreatic adenocarcinoma treated with neoadjuvant chemoradiation. Cancer 2012, 118(15):3801-3811.

39. Mierke F, Hempel S, Distler M, Aust DE, Saeger HD, Weitz J, Welsch T: Impact of Portal Vein Involvement from Pancreatic Cancer on Metastatic Pattern After Surgical Resection. Ann Surg Oncol 2016, 23(Suppl 5):730-736.

40. Rehders A, Stoecklein NH, Guray A, Riediger R, Alexander A, Knoefel WT: Vascular invasion in pancreatic cancer: tumor biology or tumor topography? Surgery 2012, 152(3 Suppl 1):S143-151.

41. John BJ, Naik P, Ironside A, Davidson BR, Fusai G, Gillmore R, Watkins J, Rahman SH: Redefining the R1 resection for pancreatic ductal adenocarcinoma: tumour lymph nodal burden and lymph node ratio are the only prognostic factors associated with survival. HPB (Oxford) 2013, 15(9):674-680.

42. Nitta T, Nakamura T, Mitsuhashi T, Asano T, Okamura K, Tsuchikawa T, Tamoto E, Murakami S, Noji T, Kurashima $Y$ et al: The impact of margin status determined by the one-millimeter rule on tumor recurrence and survival following pancreaticoduodenectomy for pancreatic ductal adenocarcinoma. Surg Today 2017, 47(4):490-497.

43. Chandrasegaram MD, Goldstein D, Simes J, Gebski V, Kench JG, Gill AJ, Samra JS, Merrett ND, Richardson AJ, Barbour AP: Meta-analysis of radical resection rates and margin assessment in pancreatic cancer. The British journal of surgery 2015, 102(12):1459-1472.

44. Neoptolemos JP, Stocken DD, Dunn JA, Almond J, Beger HG, Pederzoli P, Bassi C, Dervenis C, Fernandez-Cruz L, Lacaine F et al: Influence of resection margins on survival for patients with 
pancreatic cancer treated by adjuvant chemoradiation and/or chemotherapy in the ESPAC-1 randomized controlled trial. Annals of surgery 2001, 234(6):758-768.

45. Miyazaki M, Yoshitomi H, Shimizu H, Ohtsuka M, Yoshidome H, Furukawa K, Takayasiki T, Kuboki S, Okamura D, Suzuki D et al: Repeat pancreatectomy for pancreatic ductal cancer recurrence in the remnant pancreas after initial pancreatectomy: is it worthwhile? Surgery 2014, 155(1):58-66.

46. Kao GD, Whittington R, Coia L: Anatomy of the celiac axis and superior mesenteric artery and its significance in radiation therapy. Int J Radiat Oncol Biol Phys 1993, 25(1):131-134.

47. Dholakia AS, Kumar R, Raman SP, Moore JA, Ellsworth S, McNutt T, Laheru DA, Jaffee E, Cameron JL, Tran PT et al: Mapping patterns of local recurrence after pancreaticoduodenectomy for pancreatic adenocarcinoma: a new approach to adjuvant radiation field design. International journal of radiation oncology, biology, physics 2013, 87(5):1007-1015.

48. Yu W, Hu W, Shui Y, Zhu X, Li C, Ren X, Bai X, Yu R, Shen L, Liang T et al: Pancreatic cancer adjuvant radiotherapy target volume design: based on the postoperative local recurrence spatial location. Radiation oncology 2016, 11(1):138.

49. Goodman KA, Regine WF, Dawson LA, Ben-Josef E, Haustermans K, Bosch WR, Turian J, Abrams RA: Radiation Therapy Oncology Group consensus panel guidelines for the delineation of the clinical target volume in the postoperative treatment of pancreatic head cancer. International journal of radiation oncology, biology, physics 2012, 83(3):901-908.

50. Griffin JF, Smalley SR, Jewell W, Paradelo JC, Reymond RD, Hassanein RE, Evans RG: Patterns of failure after curative resection of pancreatic carcinoma. Cancer 1990, 66(1):56-61.

51. Kayahara M, Nagakawa T, Ohta T, Kitagawa H, Ueno K, Tajima H, Elnemr A, Miwa K: Analysis of paraaortic lymph node involvement in pancreatic carcinoma: a significant indication for surgery? Cancer 1999, 85(3):583-590.

52. Agalianos C, Gouvas N, Papaparaskeva K, Dervenis C: Positive para-aortic lymph nodes following pancreatectomy for pancreatic cancer. Systematic review and meta-analysis of impact on short term survival and association with clinicopathologic features. HPB : the official journal of the International Hepato Pancreato Biliary Association 2016, 18(8):633-641.

53. Paiella S, Sandini M, Gianotti L, Butturini G, Salvia R, Bassi C: The prognostic impact of para-aortic lymph node metastasis in pancreatic cancer: A systematic review and meta-analysis. European journal of surgical oncology : the journal of the European Society of Surgical Oncology and the British Association of Surgical Oncology 2016, 42(5):616-624.

54. Hempel S, Plodeck V, Mierke F, Distler M, Aust DE, Saeger HD, Weitz J, Welsch T: Para-aortic lymph node metastases in pancreatic cancer should not be considered a watershed for curative resection. Sci Rep 2017, 7(1):7688.

\section{Tables}

Due to technical limitations, Tables 1-3 are only available as a download in the supplemental files section 
Figures

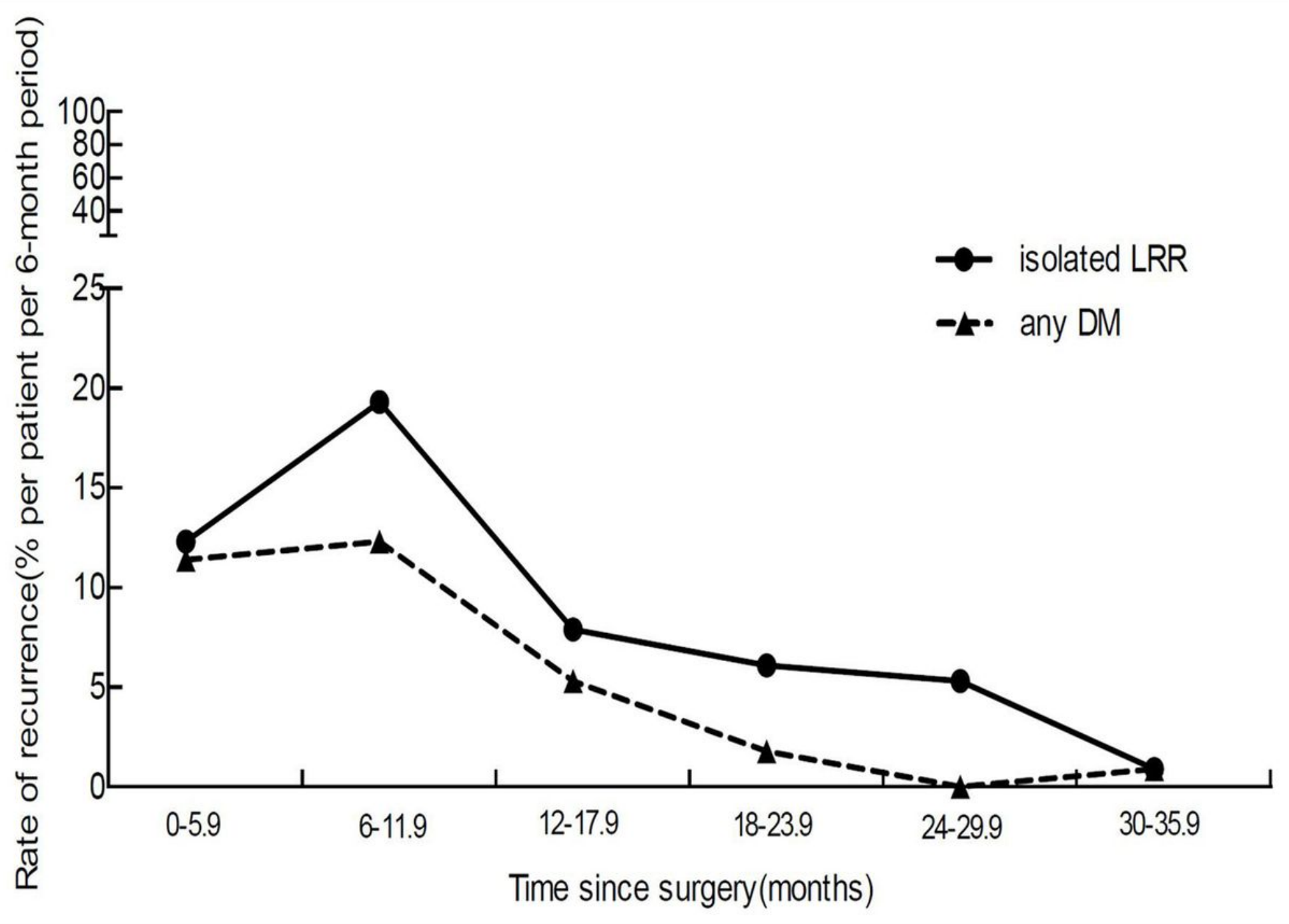

Figure 1

Rate of isolated LRR and any DM per 6-month follow-up period after surgery 


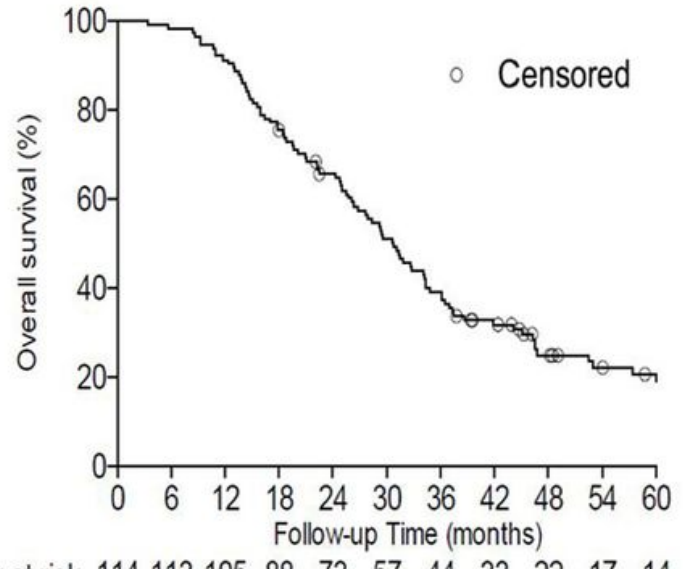

Number at risk $11411310588 \quad 73 \quad 57 \quad 44 \quad 33 \quad 22 \quad 17 \quad 14$

a

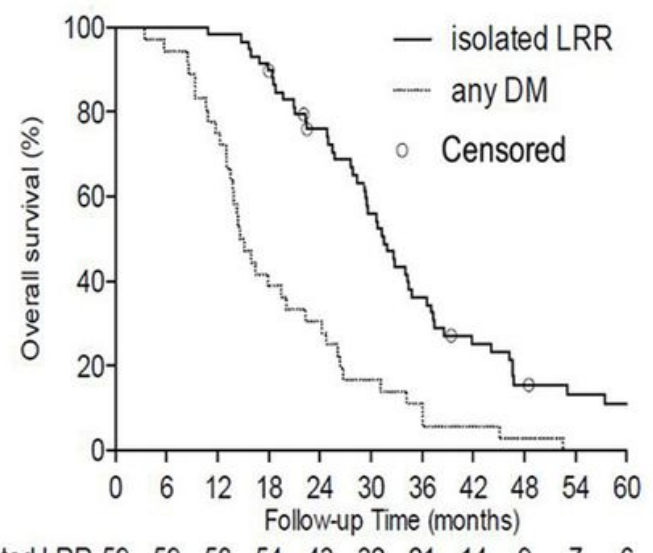

Number at risk isolated LRR $59 \begin{array}{llllllllll}59 & 58 & 54 & 43 & 32 & 21 & 14 & 9 & 7 & 6\end{array}$ $\begin{array}{lllllllllllll}\text { any M } & 35 & 34 & 27 & 15 & 12 & 7 & 5 & 4 & 2 & 1 & 1\end{array}$ c

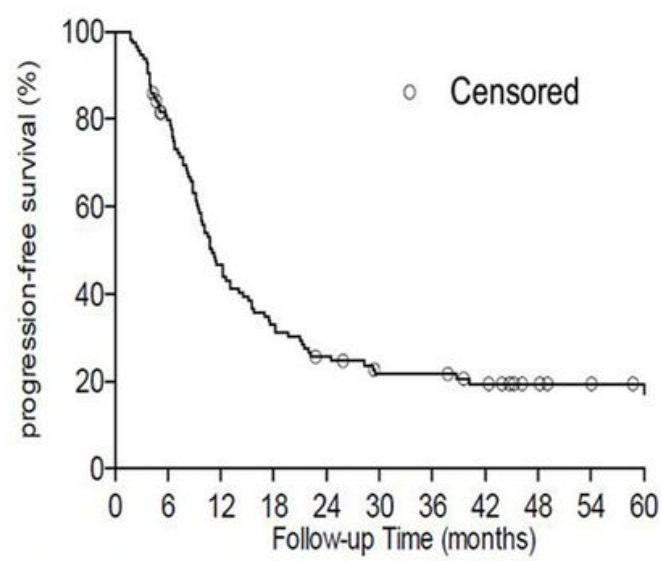

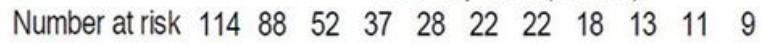

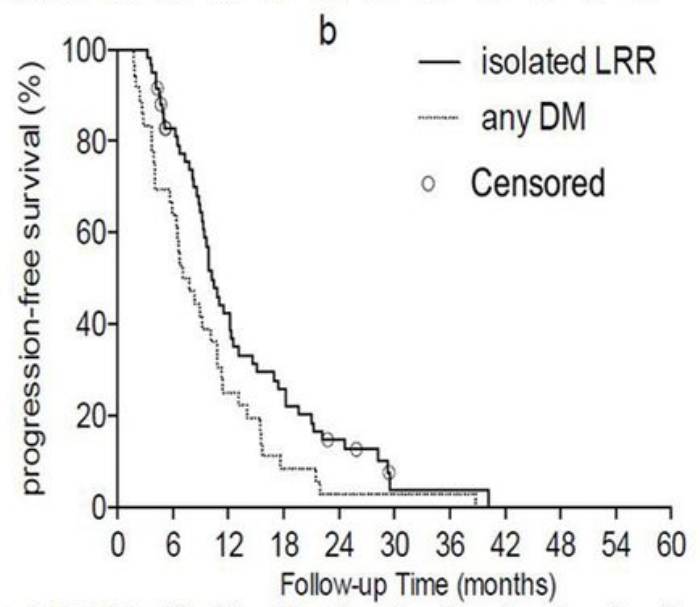

Number at risk isolated LRR $59 \begin{array}{llllllllll}46 & 24 & 15 & 8 & 2 & 2 & 1 & 0 & 0 & 0\end{array}$ any $\mathrm{M}$ $\begin{array}{llllllllllll}36 & 24 & 10 & 4 & 2 & 2 & 2 & 0 & 0 & 0 & 0\end{array}$

\section{Figure 2}

Overall and progression-free survival of patients with PDAC after adjuvant chemotherapy. (a) Overall survival of all patients $(n=114)$. (b) Progression-free survival of all patients $(n=114)$. (c) Overall survival of patients with recurrence $(n=95)$ separated into isolated LRR $(n=59)$ and any DM $(n=36)$ subgroups according to recurrence status. (d) Progression-free survival isolated LRR ( $n=59)$ compared with any DM $(n=36)$. 

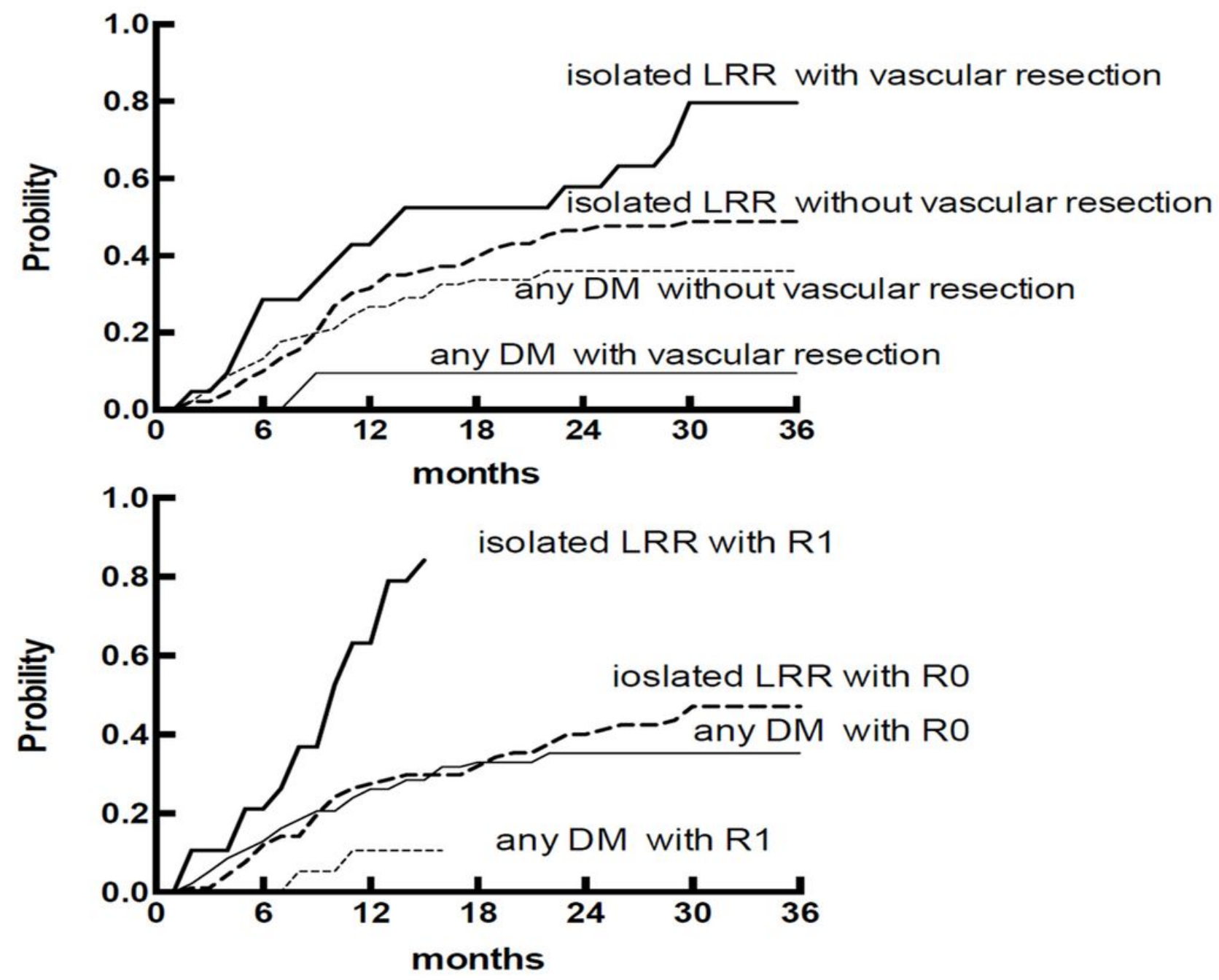

Figure 3

Predicted cumulative incidence curves of isolated LRR and any DM for resection margin status, and whether combined vascular resection 

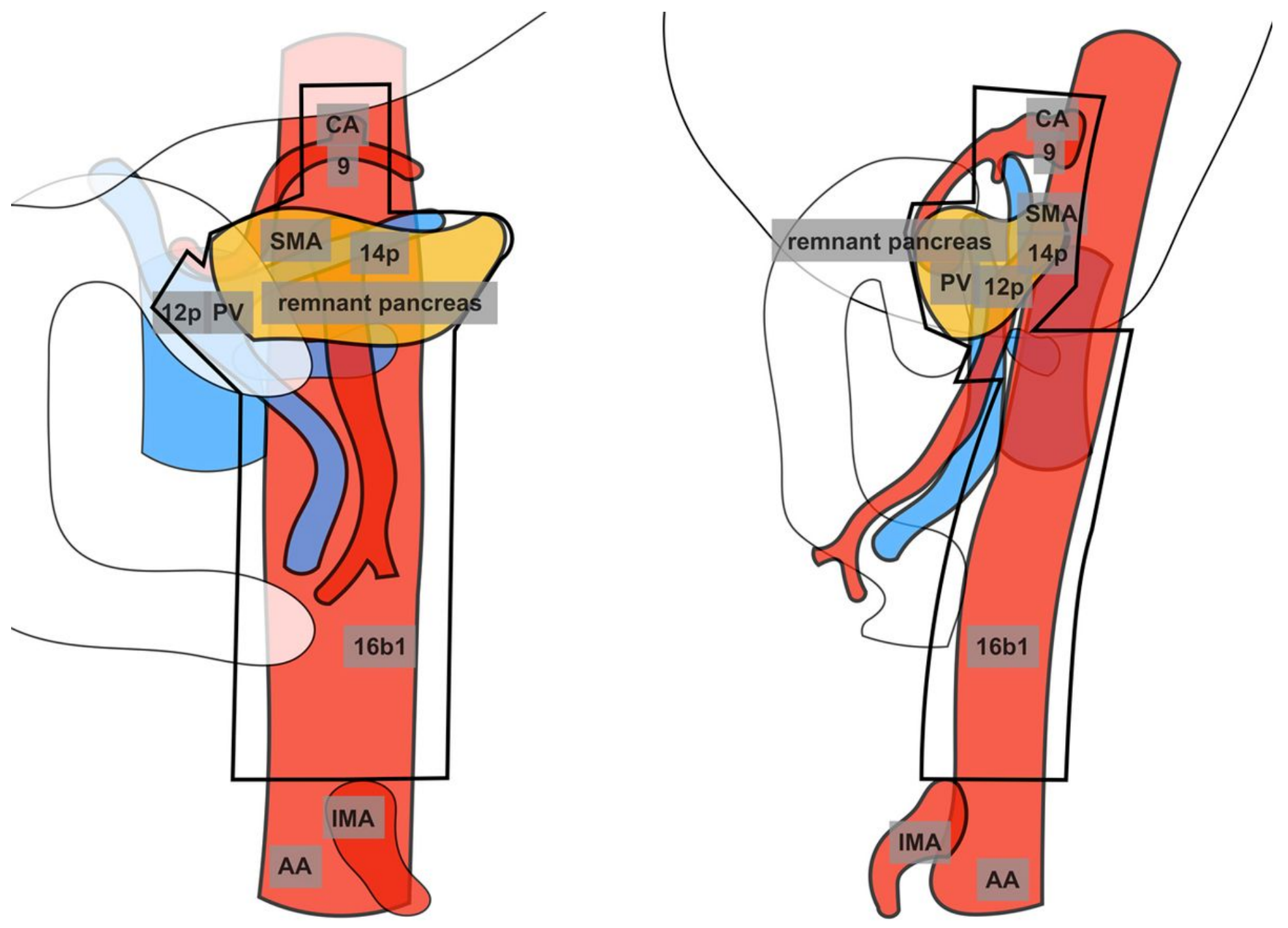

Figure 4

Suggestion of the optimal irradiation volumes design

\section{Supplementary Files}

This is a list of supplementary files associated with this preprint. Click to download.

- table2.xlsx

- table1.xlsx

- table3.xIsx 